\title{
Implantação de ferramentas da qualidade na melhoria do processo produtivo em uma panificadora
}

\author{
Implantation of quality tools in the improvement of the productive process in a bakery
}

Iasmin Constantino Barbosa do Nascimento ${ }^{1}$ (b) orcid.org/0000-0003-1173-0474

\author{
Luciana Bazante de Oliveira ${ }^{1}$ (D) orcid.org/0000-0001-9115-896X \\ 1 Pós-graduação em Gestão da Qualidade e Produtividade, Escola Politécnica de Pernambuco, Pernambuco, Brasil. \\ E-mail do autor principal: Iasmin do Nascimento iasminconstantinob@gmail.com
}

\section{Resumo}

A gestão da qualidade, ao decorrer da sua evolução, tem se tornado significativamente mais representativa e essencial à melhoria continua dos processos. Devido ao crescimento das panificadoras e a elevada normatização legal para tal segmento, tem cada vez mais aumentado a responsabilidade das empresas com a qualidade dos produtos. Este artigo foi desenvolvido para implementar soluções em uma empresa de panificação, com intuito de gerar melhorarias no processo de produção. A metodologia adotada possibilitou abordar os processos da organização, proporcionando o reconhecimento dos pontos que ocasionaram os problemas com maior relevância, gerando alternativas para obter soluções. Por meio de algumas ferramentas da qualidade: Fluxograma, Folha de Verificação, Diagrama de Ishikawa, Diagrama de Pareto e 5W2H, para melhor observar o processo, sistematizar os dados e analisar com maior clareza as principais falhas. Os dados obtidos mostraram que o principal problema eram os pães defeituosos, que foram devidamente analisado e sugerido ações de forma eficaz.

Palavras-Chave: Panificação; Produto; Defeitos; Pão; Ferramentas da qualidade.

\begin{abstract}
Quality management, as it evolves, has become significantly more representative and essential to continuous process improvement. Due to the growth of bakeries and the high legal standardization for this segment, the responsibility of companies with the quality of products is increasing. This article was developed to implement solutions in a bakery company, in order to generate improvements in the production process. The adopted methodology made it possible to approach the processes of the organization, providing the recognition of the points that caused the most relevant problems, generating alternatives to obtain solutions. Through some quality tools: Flowchart, Verification Sheet, Ishikawa Diagram, Pareto Diagram and 5W2H, to better observe the process, systematize the data and analyze more clearly the main flaws. The data obtained showed that the main problem was the defective breads, which was properly analyzed and solved effectively.
\end{abstract}

Key-words: Baking; Product; Defects; Bread; Quality tools. 


\section{Introdução}

As ferramentas da qualidade podem ser utilizadas para identificar defeitos e não conformidades geradas durante o processo produtivo e investigar suas possíveis causas, com a finalidade de estudar alternativas para eliminá-las ou amenizá-las [1].

De acordo com a Associação Brasileira da Indústria de Panificação (ABIP), em 2018 o Brasil apresentou cerca de 70520 padarias. As padarias são caracterizadas na maioria das vezes, como pequenas empresas que trabalham em sua pluralidade com vendas de pães e produto de confeitaria [2]. Com as exigências do mercado crescendo cada vez mais, a empresa que deseja obter bons resultados deve sempre buscar e considerar aspectos básicos que garantam a sobrevivência da organização no mercado atual, os principais são: competitividade da empresa e qualidade dos produtos, sendo nesse caso $2 \mathbf{s} 1$ ferramentas da qualidade a escolha para promover a melhoria de tais resultados [3].

A panificadora abordada nesse trabalho existe há 25 anos e possui 20 funcionários em revezamento nos turnos da manhã e tarde. Ela fabrica uma grande variedade de pães, bolos e salgados. A qualidade dos seus produtos tornou-se algo indispensável e a empresa está constantemente inovando para manterse no mercado com estabilidade, investindo ainda mais em capacitação dos seus funcionários, disponibilizando diversos treinamentos, melhorando os seus processos e promovendo novas tecnologias para o setor [4].

Com isso, foi possível retratar a importância da aplicação das ferramentas da qualidade para a melhoria dos resultados das organizações, por meio da aplicação de algumas delas no processo de fabricação de pães.

\section{Metodologia}

Apesar de um alto mix de produtos, a intervenção apresentada nesse trabalho foi aplicada no item do pão francês, produto mais vendido e carro-chefe da empresa, cuja quantidade produzida é, em média, de 1.915 unidades por dia.

As ferramentas da qualidade foram usadas desde a escolha, identificação e caracterização do problema e, também, na sua solução. Por meio da coleta de dados, foi possível a identificação de possíveis causas para as não conformidades, possibilitando a obtenção de melhorias.

89
As informações foram reunidas por meio de folha de verificação devido ao alto índice de reclamações dos clientes de pães fora do padrão, com isso foram analisadas algumas amostras de pão francês, para a identificação de não conformidades que apresentaram maior frequência, tais como: pão tostado, pão deformado, pão com baixa quantidade de fermento e pão dormido.

As amostras foram analisadas na panificadora no período de 07/01/2019 a 19/01/2019, de segunda à sábado. Por meio das informações encontradas, foi viável a execução das ferramentas da qualidade citadas anteriormente e a identificação de possíveis causas das não conformidades. Através dos resultados será possível indicar melhorias para a fabricação.

\section{Revisão de Literatura}

\subsection{Ferramentas da Qualidade}

As ferramentas da qualidade disponibilizam um apoio indispensável à revisão e melhoria dos processos. Dispõe de dados essenciais cada ferramenta que em grupo complementam a metodologia [5].

São utilizadas para medir, determinar, verificar e indicar meios para solucionar desvios que afetam o desenvolvimento dos processos de produção. Proporcionam um maior controle dos processos ou melhorias na tomada de decisões [6].

O uso das ferramentas é de extrema importância para os sistemas de gestão, formando um grupo de ferramentas para aplicação na melhoria de produtos, serviços e processos. A seguir, as ferramentas aplicadas neste trabalho acadêmico:

\subsubsection{Fluxograma}

É uma ferramenta de fácil aplicação, que proporciona excelentes vantagens, associadas ao reconhecimento de falhas no fluxo do processo e identificação de momentos ociosos [7].

Para conquistar uma interpretação exata da produção é preciso realizar anotação de todas as etapas do processo. A confirmação do fluxograma é realizada através de símbolos uniformizados em sua maior parte, que ajudam a verificação do fluxo do processo da panificadora [8].

DOI: 10.xxxx/s11468-014-9759-3 


\subsubsection{Folha de Verificação}

A folha de verificação é uma ferramenta aplicada com propósito de demonstrar as informações por um meio compreensível e preciso, além de diminuir falhas e complicações no momento da extração dos dados e assegurar que estas informações sejam confiáveis [9].

Os principais fundamentos para a aplicação da folha de verificação são a sistematização imparcial das informações de quem efetua as coletas, a arrumação das informações do decorrer da análise, evitando a obrigação de organizar posteriormente e a simplificação da pesquisa de informações [10].

\subsubsection{Diagrama de Pareto}

O Diagrama de Pareto é composto por um gráfico de barras onde elas são classificadas com base na mais alta até a mais baixa ocorrência e é marcada uma curva que demonstra os percentuais reunidos de cada barra [11].

É uma forte ferramenta para ajudar no entendimento e priorização da abordagem dos problemas, com as principais causas visualizadas do lado esquerdo do diagrama, e as causas menores apresentadas em ordem decrescente, ao lado direito [12].

\subsubsection{Diagrama de causa e efeito}

O Diagrama de causa e efeito é formado por um conjunto de pessoas focadas a pesquisar e reconhecer as fundamentais causas produzidas de um problema encontrado [13].

Esta ferramenta tem como propósito encontrar as supostas razões das não conformidades, proporcionando seu embate e, portanto, melhoria da qualidade dos processos. Na atualidade o diagrama não é somente aplicado para resolver as características da qualidade do produto, como também em outros campos [14].

\section{$3.1 .5 \quad 5 W 2 H$}

Esta ferramenta é representada por sete perguntas específicas, que são:

- What? (O que?)

- Who? (Quem?)

- Where? (Onde?)

- When? (Quando)

- Why?(Por quê?)
- How? (Como?)

- How much? (Quanto Custa?)

Esta ferramenta é um plano de ação para práticas pré-estabelecidas que precisem ser desenvolvidas com a maior clareza possível, além de funcionar como um mapeamento dessas atividades [15].

É bastante aplicada em diversos segmentos, devido ao seu entendimento e facilidade de aplicação [16].

\subsection{Setor de panificação}

Por causa do constante desenvolvimento do setor de panificação, os empreendedores perceberam a necessidade de comercializar além de pão, produtos diversificados [17].

O setor de panificadoras e confeitarias movimenta R\$ 92 bilhões de faturamento, cerca de $70 \%$ foi resultado de produção própria Com o seu desenvolvimento, tornou-se a segunda maior rede de distribuição de alimentos, só ficando atrás dos supermercados [18].

Segundo a Associação Brasileira da Indústria da Panificação e Confeitaria (ABIP), em 2018 o setor acarretou mais de 2 milhões de funções de trabalho, referindo-se a 890 mil empregos diretos e 1,2 milhões indiretos.

Devido a todo esse desenvolvimento, o setor necessita de muitas melhorias, sendo ainda possível localizar muitos desvios que impactam na qualidade do pão, que resulta principalmente nas imperfeições no processo produtivo e da maneira como são manipulados os equipamentos [19].

\section{Implantação e resultados}

Com a construção do fluxograma apresentado na Figura 1 foi possível analisar o fluxo da produção. Compreender o processo é um passo necessário para adequá-lo. 


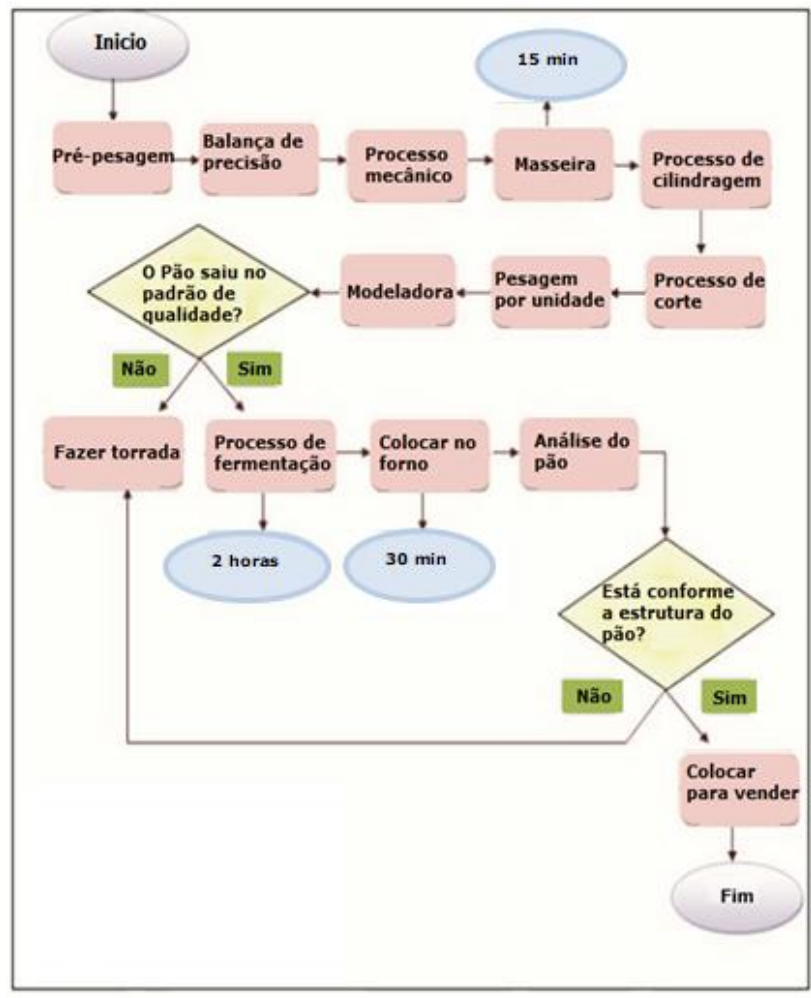

Figura 1: Fluxograma

Fonte: Autor (2019)

O mapeamento mostrado é referente ao fluxograma com as suas devidas correções, visando evitar desperdícios e gastos com os pães defeituosos, o meio encontrado por intermédio desta ferramenta foi utilizar os pães com variação de formato para produção de torradas.

Para localizar as possíveis causas dos problemas, foi aplicada a folha de verificação com a finalidade de relatar o tempo estimado das amostras na masseira. Este fator é determinante para textura da massa e consequentemente a quantidade de pães defeituosos.

Observou-se que a folha de verificação foi de extrema relevância para a obtenção de dados a serem organizados de forma estruturada. Além de auxiliar como base para aplicabilidade de outras ferramentas, onde obteve os resultados indicados na Tabela 1.
Tabela 1: Folha de Verificação do tempo na masseira

\begin{tabular}{|c|c|c|c|c|}
\hline Amostra & Hora & $\begin{array}{c}\text { Tempo na masseira } \\
\text { (min) }\end{array}$ & $\begin{array}{c}\text { Itens } \\
\text { defeituosos }\end{array}$ & Observặ̃o \\
\hline 1 & 4:00 & 10 & 8 & Textura fora dos limites de toler ância. \\
\hline 2 & $4: 20$ & 12 & 5 & Textura fora dos limites de toler ância. \\
\hline 3 & $4: 35$ & 14 & 2 & \\
\hline 4 & $4: 50$ & 16 & 0 & \\
\hline 5 & $5: 20$ & 14 & 2 & \\
\hline 6 & $5: 40$ & 16 & 1 & \\
\hline 7 & 6:00 & 15 & 0 & \\
\hline 8 & 6:30 & 17 & 3 & Textura fora dos limites de toler ância. \\
\hline 9 & 7:00 & 15 & 0 & \\
\hline 10 & $7: 40$ & 16 & 0 & \\
\hline 11 & $8: 00$ & 11 & 6 & Textura for a dos limites de toler ância. \\
\hline 12 & $8: 15$ & 10 & 8 & Textura fora dos limites de toler ância. \\
\hline 13 & $8: 30$ & 12 & 6 & Textura fora dos limites de toler ância. \\
\hline 14 & $8: 50$ & 9 & 8 & Textura fora dos limites de toler ância. \\
\hline 15 & 9:00 & 8 & 10 & Textura fora dos limites de toler ância. \\
\hline 16 & $9: 20$ & 10 & 8 & Textura fora dos limites de tolerância. \\
\hline 17 & $9: 40$ & 12 & 5 & Textura fora dos limites de toler ância. \\
\hline 18 & $10: 00$ & 15 & 0 & \\
\hline 19 & $10: 20$ & 14 & 1 & \\
\hline 20 & $10: 40$ & 16 & 1 & \\
\hline \multicolumn{2}{|c|}{ Média Arnustual } & 13,1 & 3,7 & \\
\hline
\end{tabular}

Fonte: Autor (2019)

Verificou-se que o tempo na masseira foi relevante para a produção da massa, por meio da folha de verificação foi possível observar que quanto menor o tempo na masseira maior o índice de pães defeituosos, visto que a massa tem que adquirir consistência firme e de bom preparo, pois quanto mais próximo do tempo padrão de 15 min mais consistente será a massa. Isso significa que, para tempos curtos ou prolongados na masseira, há uma maior chance de pães apresentarem textura fora dos limites de tolerância.

$\mathrm{Na}$ etapa a seguir do processo é levar as massas dos pães ao processo de fermentação por 2 horas, atingindo o tamanho esperado. Em seguida são levadas até o forno, para assar por $30 \mathrm{~min}$ a $180^{\circ} \mathrm{C}$.

Alguns pães saem com aspectos não padronizados. Um dos fatores que pode acarretar essa não conformidade é a baixa quantidade fermento encontrado nos pães, que influencia no crescimento das massas. Outra evidência encontrada na produção é a elevada temperatura do forno, gerando pães fora do padrão, onde ambos são ocasionados pelos responsáveis da produção.

A seguir na Tabela 2 é possível verificar o número de não conformidades ocorridas nos 12 dias de análise.

Tabela 2: Dados de não conformidades 


\begin{tabular}{|c|c|c|}
\hline Dia & Quantidade & Não conformidade \\
\hline \multirow[t]{4}{*}{1} & 9 & Tostado \\
\hline & 3 & Deformado \\
\hline & 1 & Pouco Fermento \\
\hline & 2 & Cascudo \\
\hline \multirow[t]{4}{*}{2} & 3 & Tostado \\
\hline & 2 & Deformado \\
\hline & 1 & Pouco Fermento \\
\hline & 2 & Cascudo \\
\hline \multirow[t]{4}{*}{3} & 6 & Tostado \\
\hline & 4 & Deformado \\
\hline & 0 & Pouco Fermento \\
\hline & 2 & Cascudo \\
\hline \multirow[t]{4}{*}{4} & 4 & Tostado \\
\hline & 3 & Deformado \\
\hline & 1 & Pouco Fermento \\
\hline & 1 & Cascudo \\
\hline \multirow[t]{4}{*}{5} & 3 & Tostado \\
\hline & 5 & Deformado \\
\hline & 2 & Pouco Fermento \\
\hline & 3 & Cascudo \\
\hline \multirow[t]{4}{*}{6} & 2 & Tostado \\
\hline & 1 & Deformado \\
\hline & 3 & Pouco Fermento \\
\hline & 2 & Cascudo \\
\hline \multirow[t]{4}{*}{7} & 2 & Tostado \\
\hline & 5 & Deformado \\
\hline & 2 & Pouco Fermento \\
\hline & 4 & Cascudo \\
\hline \multirow[t]{4}{*}{8} & 3 & Tostado \\
\hline & 2 & Deformado \\
\hline & 2 & Pouco Fermento \\
\hline & 1 & Cascudo \\
\hline \multirow[t]{4}{*}{9} & 1 & Tostado \\
\hline & 2 & Deformado \\
\hline & 2 & Pouco Fermento \\
\hline & 1 & Cascudo \\
\hline \multirow[t]{4}{*}{10} & 4 & Tostado \\
\hline & 5 & Deformado \\
\hline & 2 & Pouco Fermento \\
\hline & 3 & Cascudo \\
\hline \multirow[t]{4}{*}{11} & 1 & Tostado \\
\hline & 3 & Deformado \\
\hline & 1 & Pouco Fermento \\
\hline & 3 & Cascudo \\
\hline \multirow[t]{4}{*}{12} & 1 & Tostado \\
\hline & 3 & Deformado \\
\hline & 1 & Pouco Fermento \\
\hline & 2 & Cascudo \\
\hline
\end{tabular}

Fonte: Autor (2019)

Observou-se que a quantidade de não conformidades segue uma distribuição irregular em função do dia. Foi perceptível que ocorre um maior número de pães defeituosos no primeiro dia da análise, conforme pode ser visualizado na Figura 2, 0 que foi atribuído à maior demanda dos consumidores e à sobrecarga dos funcionários.

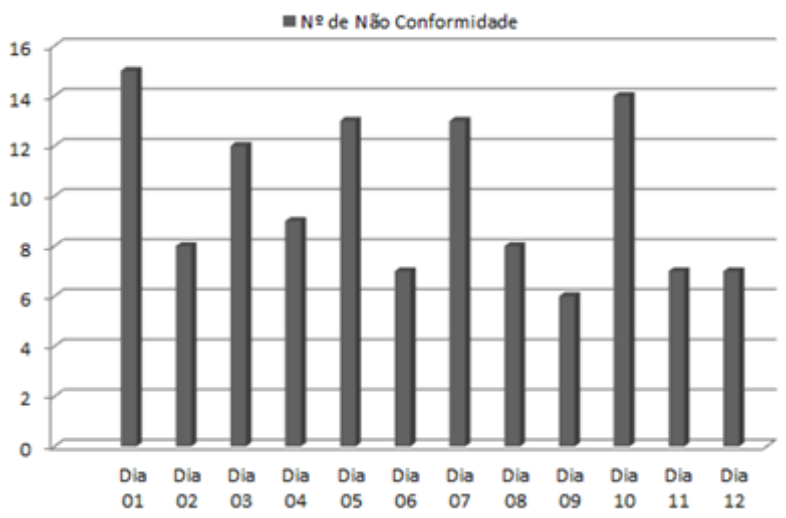

Figura 2: $\mathrm{N}^{\circ}$ de não conformidades em 12 dias Fonte: Autor (2019)

O dia da semana onde ocorreram mais variações na produção do pão foi a segunda-feira, devido a maior procura da clientela e maior demanda por produtos.

No dia que apresentou maior relevância foi possível observar que no dia anterior a panificadora não apresentou expediente, com isso ocorreu um acumulo de tarefas.

Fazendo uma análise dos turnos no primeiro dia, o turno da manhã se sobrepôs aos demais em números de não conformidade, com isso foi realizada uma estratificação, distribuída por turnos, como observado na Figura 3.

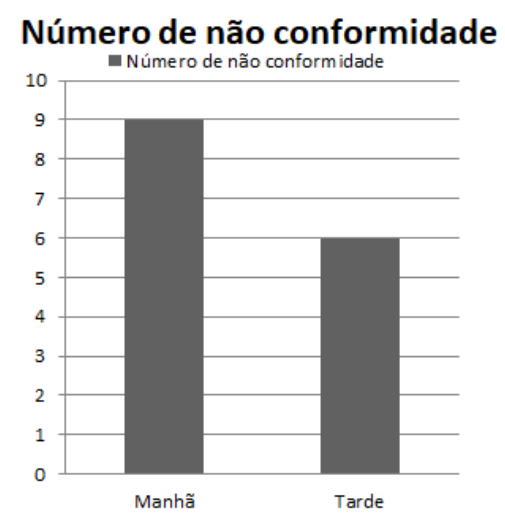

Figura 3: Dados dos turnos.

Fonte: Autor (2019)

No primeiro dia de análise, que foi realizada no turno da manhã, foi possível observar que as não conformidades foram mais frequentes, devido à redução do número de funcionários por motivo de faltas na produção, bem como o aumento dos pedidos. Por meio desses dados, foi possível aplicar outra ferramenta demonstrada a seguir.

Os defeitos que apresentam maior representatividade são, respectivamente, deformados, cascudo, tostado e pouco fermento. Portanto, a análise através do gráfico de Pareto 
proporcionou identificar o item defeituoso com maior ênfase no processo produtivo, como pode ser observado na Figura 4.

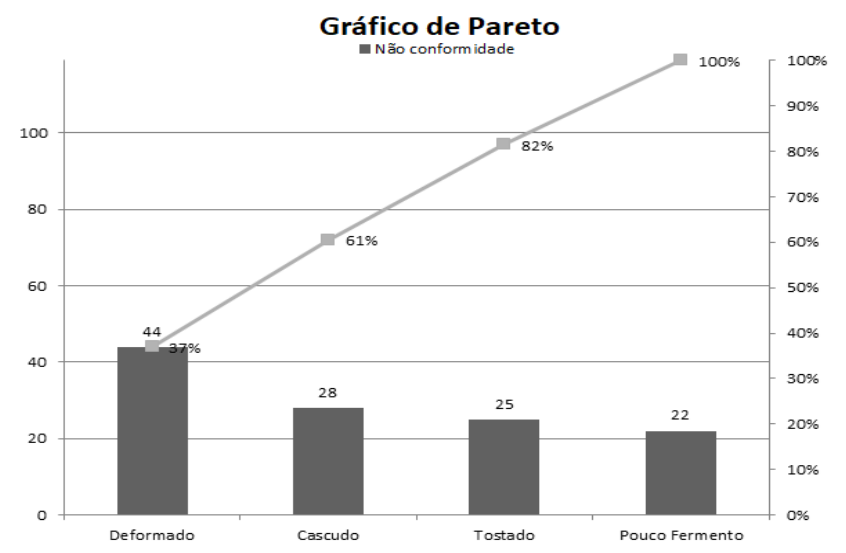

Figura 4: Gráfico de Pareto Fonte: Autor (2019)

Nesta etapa, foi possível determinar que a falta de uma orientação de trabalho para determinar os parâmetros de funcionamento do processo, por exemplo, o tempo exato que deve permanecer na máquina e a baixa velocidade para a mistura da massa ficar homogênea, são fatores importantes que podem interferir na qualidade da massa para produção do produto, com isso, necessitam de uma modificação rápida, na Figura 5.

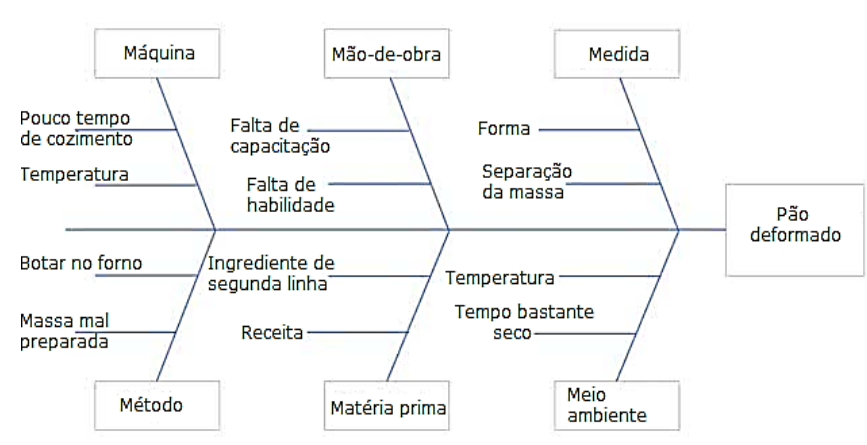

Figura 5: Diagrama de Causa e Efeito.

Fonte: Autor (2019)

Ao verificar o diagrama de causa e efeito é possível observar que um dos problemas é a massa mal preparada, pois alguns funcionários não seguem as medidas de preparo do pão francês, acarretando pães defeituosos, fora do padrão desejado e gerando o aumento de desperdício.
Outro problema importante é a falta de capacitação dos funcionários, que recebem tarefas não enquadradas no perfil dos seus cargos.

Verificou-se como relevante a carência de maquinários regulados, com isso proporcionando desperdícios ocasionados pela produção de má qualidade, todos esses fatores podem ser verificados no Anexo A.

Posterior à análise da produção do pão francês, foram percebidas melhorias que foram adotadas pela panificadora.

A primeira iniciativa de melhoria foi a reunião com os funcionários da produção para pedir maior atenção, na hora da manipulação do fermento para fabricação do pão francês, visto que tem interferido na qualidade do produto final. Foi uma forma de orientar os funcionários para que os mesmos tenham um melhor desempenho e sigam 0 formato padrão da panificadora.

A segunda atitude de melhoria foi à diminuição do produto no forno, onde o colaborador responsável pelo maquinário tem a função de regular a temperatura quando necessário, visto que foi passado instruções durante a capacitação do funcionário da produção.

A terceira ação de melhoria promoveu o aumento da qualidade do produto, onde as trocas dos ingredientes por outros de melhor qualidade trouxe benefícios tanto para o processo, quanto para lucratividade da panificadora.

A quarta conduta realizada pelo proprietário foi a instalação dos ventiladores, ocasionando maior bem estar aos funcionários, com isso gerando uma melhor qualidade do desempenho dos mesmos.

A quinta proposta de ação sugerida ao proprietário foi a de expandir o quadro de colaboradores na produção do processo, consequentemente reduzindo a sobrecarga dos funcionários.

\section{Conclusão}

No presente artigo foi possível ter uma perspectiva mais ampla da panificadora abordada, onde foi estudado o processo do pão francês, analisada a satisfação dos funcionários no ambiente interno, verificando os principais problemas e suas soluções.

Dessa maneira, os problemas com maiores impactos no processo de produção foram manipulação do fermento, tempo na masseira, tempo de cozimento, capacitação dos funcionários encontrados no diagrama de causa e efeito. 
Uma vez que o problema de pães deformados foi determinado com maior índice de defeitos, foram executadas algumas medidas para solucioná-lo, tais como: os produtos de qualidade, a correta manipulação do fermento, marcação ideal no tempo da masseira e regulagem do forno.

Após todas essas ações, foi possível observar que ocorreram melhorias significativas no processo do pão francês, proporcionando uma redução significativa dos desperdícios defeitos.

Outro ponto relevante solucionado foi a temperatura do ambiente, proporcionando um local de trabalho favorável aos colaboradores, com isso aumentando seus desempenhos na produção.

As ferramentas de qualidades selecionadas foram de acessível aplicação, pois são ajustáveis a qualquer empresa. Através da sua aplicação sistemática, a panificadora obteve otimização dos processos e redução das não conformidades, elevando a rentabilidade do negócio. Desta forma, as ferramentas da qualidade contribuíram para um melhor desempenho dos colaboradores, das atividades e maior satisfação dos clientes. Após a implantação de todas essas ferramentas, a panificadora vem apresentando melhorias nos seus resultados, visto que as mudanças foram satisfatórias para uma produção mais eficaz.

\section{Referências}

[1] DEMING, W. Edwards. Qualidade: A revolução da Administração, 1990. Marques Saraiva, Rio de Janeiro: 1982;

[2] SHEWHART, W. A. Economic control of quality of manufactured products. São Paulo: ASQPR, 1986;

[3] FREITAS, Kaliany Dias de. Aplicação das ferramentas da qualidade em uma panificadora como método de melhoria do processo produtivo: Estudo de caso. XXXIV Encontro Nacional De Engenharia De Produção. Curitiba-PR, 2014;

[4] PALADINI, E. P. Avaliação estratégica da qualidade. São Paulo: Atlas 2002;

[5] Dados da Associação Brasileira da Indústria de Panificação - ABIP. Disponível em: $<$ http://www.abip.org.br/perfil.aspx> Acessado em: 29 dez. de 2018;

[6] WERKEMA, M. C. C. As ferramentas da Qualidade no Gerenciamento de Processos. Belo Horizonte: Editora de Desenvolvimento Gerencial,1995.
[7] WERKEMA, M. C. C. Ferramentas estatísticas básicas para o gerenciamento de processos. Belo Horizonte: Werkema Editora Ltda,2006;

[8] SAMOHYL, Robert W. Controle estatístico da qualidade. $5^{\circ}$ ed. Rio de Janeiro: Elsevier, 2009;

[9] BITTENCOURT, Helio. Controle Estatístico da Qualidade. Porto Alegre: 2014.

[10] MOURA, L. R. Qualidade Simplesmente. Total. Rio de Janeiro: Qualitymark, 2003;

[11] VIEIRA, S. Estatística para a Qualidade: como avaliar com precisão a qualidade em produtos e serviços. Rio de Janeiro: Editora Campus, 2012.

[12] GIOCONDO. Francisco I. César. Ferramentas da Qualidade: Instrumentos para gerenciamento de processos e melhoria contínua. São Paulo, 2011;

[13] OAKLAND, J. Gerenciamento da qualidade total. São Paulo: Nobel, 1994;

[14] SAMOHYL, R. W. Controle Estatístico da Qualidade. Rio de Janeiro: Campus, 2009;

[15] SILVA, A.RORATTOO, L.SERVAT, M.DORNELES, L.POLACINSK, E. Gestão da qualidade: Aplicação da ferramenta $5 \mathrm{w} 2 \mathrm{~h}$ como plano de ação para projeto de abertura de uma empresa. Horizontina-RS: SIEF, 2013;

[16] DRUCKER, Peter F. Desafios gerenciais para século XXI. São Paulo: Thomsom, 2001;

[17] GALUCH, L. Modelo para Implementação das Ferramentas Básicas do Controle Estatístico de Processo. Dissertação (Mestrado em Engenharia de Produção) - Programa de Pós-Graduação em Engenharia de Produção, Florianópolis, Universidade Federal de Santa Catarina, 2002;

[18] ABIP; ITPC. Performance do setor de panificação e confeitaria brasileiro em 2014. 2015. Acessado em: 19 de abril 2019;

[19] SOUZA, Mariana de Albuquerque e. Boas práticas para Padarias e Confeitarias. Porto Alegre, 2012. Acessado em: 19 de abril 2019; 


\section{ANEXo A: PLANO de AÇÃo}

\begin{tabular}{|c|c|c|c|c|c|c|c|}
\hline Itens & $\begin{array}{l}\text { What? } \\
\text { o Que? }\end{array}$ & $\begin{array}{l}\text { When? } \\
\text { Quando? }\end{array}$ & $\begin{array}{l}\text { Where? } \\
\text { Onde? }\end{array}$ & $\begin{array}{l}\text { Why? } \\
\text { Porque? }\end{array}$ & $\begin{array}{l}\text { Who? } \\
\text { Quem? }\end{array}$ & $\begin{array}{l}\text { How? } \\
\text { Como? }\end{array}$ & $\begin{array}{l}\text { How Much? } \\
\text { Quanto? }\end{array}$ \\
\hline $\begin{array}{l}\text { Falta de capacitação e } \\
\text { Falta de Habilidade }\end{array}$ & $\begin{array}{l}\text { Procurar o Sindicato para } \\
\text { proporcionar palestras e } \\
\text { cursos para área de } \\
\text { aperfeiçoamento }\end{array}$ & $11 / 01 / 2019$ & $\begin{array}{c}\text { Nas instalações do } \\
\text { Sindicato }\end{array}$ & $\begin{array}{l}\text { Os cursos irão } \\
\text { melhorar a } \\
\text { qualidade da } \\
\text { produção do pão } \\
\text { e valorização do } \\
\text { funcionário }\end{array}$ & Proprietário & $\begin{array}{c}\text { Após a parceria, será feito um } \\
\text { calendário de como serão os } \\
\text { cursos }\end{array}$ & $\begin{array}{l}\text { Custo não } \\
\text { informado }\end{array}$ \\
\hline Temperatura & Climatizar o ambiente & $09 / 01 / 2019$ & $\begin{array}{l}\text { No setor de } \\
\text { produção da } \\
\text { panificação }\end{array}$ & Está muito calor & $\begin{array}{c}\text { Proprietário e } \\
\text { empresa } \\
\text { especializada }\end{array}$ & $\begin{array}{l}\text { Sócio proprietário realizou a } \\
\text { cotação dos ventiladores e do } \\
\text { serviço. Comprou.,os materiais } \\
\text { e contratou o serviço. }\end{array}$ & 1957,00 \\
\hline $\begin{array}{c}\text { Deformidade e } \\
\text { coloração não uniforme } \\
\text { do pão }\end{array}$ & $\begin{array}{l}\text { Defeito na máquina } \\
\text { modeladora e forno } \\
\text { desregulado }\end{array}$ & $14 / 01 / 2019$ & Assistência técnica & $\begin{array}{l}\text { Para solucionar o } \\
\text { problema }\end{array}$ & $\begin{array}{l}\text { Técnico da } \\
\text { área }\end{array}$ & $\begin{array}{l}\text { Processo de manutenção } \\
\text { corretiva }\end{array}$ & $\begin{array}{l}\text { Custo não } \\
\text { informado }\end{array}$ \\
\hline Tempo de Cozimento & $\begin{array}{l}\text { Aumentar a temperatura } \\
\text { do forno }\end{array}$ & $\begin{array}{l}\text { Em curto } \\
\text { prazo }\end{array}$ & $\begin{array}{l}\text { No setor de } \\
\text { produção da } \\
\text { panificação }\end{array}$ & $\begin{array}{l}\text { Diminuiçãa da } \\
\text { duraçãa do } \\
\text { processo }\end{array}$ & $\begin{array}{l}\text { Funcionários } \\
\text { da produção }\end{array}$ & Aumentar a potência do forno & $\begin{array}{l}\text { Aumento de } \\
0,5 \% \text { do custo } \\
\text { operacional }\end{array}$ \\
\hline $\begin{array}{l}\text { Quantidade de } \\
\text { funcionário }\end{array}$ & $\begin{array}{l}\text { Contratação de no mínimo } \\
\text { mais um funcionário. }\end{array}$ & $\begin{array}{l}\text { Previsã̃o } \\
\text { futura }\end{array}$ & Panificação & $\begin{array}{l}\text { O funcionário } \\
\text { não está } \\
\text { conseguindo } \\
\text { realizar seu } \\
\text { trabalho por } \\
\text { acumulo de } \\
\text { funcões. }\end{array}$ & Proprietário & $\begin{array}{c}\text { Será aberta uma vaga para que } \\
\text { possa ser feito a seleção. }\end{array}$ & $\begin{array}{l}1 \text { salário }+ \\
\text { benefícios }\end{array}$ \\
\hline $\begin{array}{c}\text { Ingrediente de segunda } \\
\text { linha }\end{array}$ & Produtos de segunda linha & $\begin{array}{l}\text { Em curto } \\
\text { prazo }\end{array}$ & $\begin{array}{l}\text { Panificação e nos } \\
\text { fornecedores }\end{array}$ & $\begin{array}{c}\text { Melhorar o sabor } \\
\text { do pão }\end{array}$ & $\begin{array}{c}\text { Novos } \\
\text { fornecedores }\end{array}$ & $\begin{array}{l}\text { Aumentar a qualidade do } \\
\text { produto }\end{array}$ & $\begin{array}{l}\text { Custo não } \\
\text { informado }\end{array}$ \\
\hline
\end{tabular}

Fonte: Autor (2019) 